\title{
HUNTER - HYBRID UNIFIED TRACKING ENVIRONMENT
}

\author{
Aislan Gomide Foina and Francisco Javier Ramirez-Fernandez \\ Universidade de São Paulo \\ Brazil
}

\section{Introduction}

Pervasive computing is an emerging paradigm used nowadays for very distinct applications. It is a model of human-computer interaction in which computer devices are embedded in an environment, exchanging information between themselves and the human preset as well. It has been called Everyware (Greenfied, 2006). Some of its application can go from smart homes for elderly people, to automatic toll billing system for vehicles $(\mathrm{Xu}, 2008)$. In many cases, pervasive applications make use of standard technology called Radio Frequency Identification - RFID. RFID is an identification technology similar to barcode, but instead of using laser beams and optical cameras to detect a printed code in a label, it uses radio waves to communicate with a microchip built-in this same label and gets its identification wirelessly. Its application can vary as much as the pervasive computing application.

In this book chapter, a case study will be explored using pervasive computing principles and RFID technology to supervise a bulk cargo unloading process in an automated way to reduce fraud attempts and to increase the efficiency of the unloading place.

Bulk cargo is a very common transport modality for ship commodities. Large bulk wagons and bulk ships can convey hundreds of thousand tons of loads. Thus, this modality is used mostly by railway and port authorities.

A regular bulk load process consists of three tasks: 1) measurement of the weight without the product - empty weight; 2) the load of the product in the transporter; and 3) the measurement of the weight with the product loaded - full weight. The unload process is similar to the load processes, but in the reverse order, as seen in Fig. 1: 1) Full weight measurement; 2) cargo unload; and 3) empty weight measurement. The difference between the first and the last weight measurements is the weight of the product loaded or dropped.

There are two major types of trucks that convey bulk cargo: dump truck and bulk truck. The first type has a hydraulic system to tip the trailer, dumping the product by the action of gravity. The second type does not have this mechanism, so it depends on a hydraulic tipping bridge to tip the whole truck. Fig. 2. shows both types of truck unloading the cargo.

A great problem faced by the companies that work with this modality of cargo is theft. A common theft reported by the company studied is trailer exchange. A truck full of a product arrives at the unload area and it performs the full weight task normally. After that, this 
truck goes to an outside area, exchanges the full trailer for an empty one, and the driver defrauds the documents. Later, it goes back to do the empty weight task and leaves. In brief, the company pays for the non-received product, and the truck driver sells this cargo to another company, getting the money two times for only one cargo. This theft is called as full load theft and, according to the company, it represents $90 \%$ of the total loss of the company with stolen cargo.

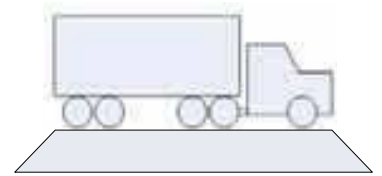

\section{Full Weight} Capture

Total Weight $=$ truck + cargo

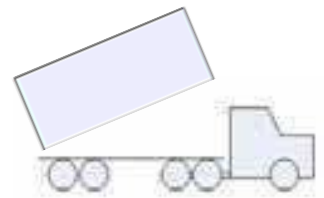

Unload

Cargo dropped

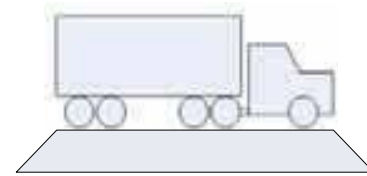

\section{Empty Weight Capture}

Total Weight = truck

Fig. 1. The three tasks of the unload process.

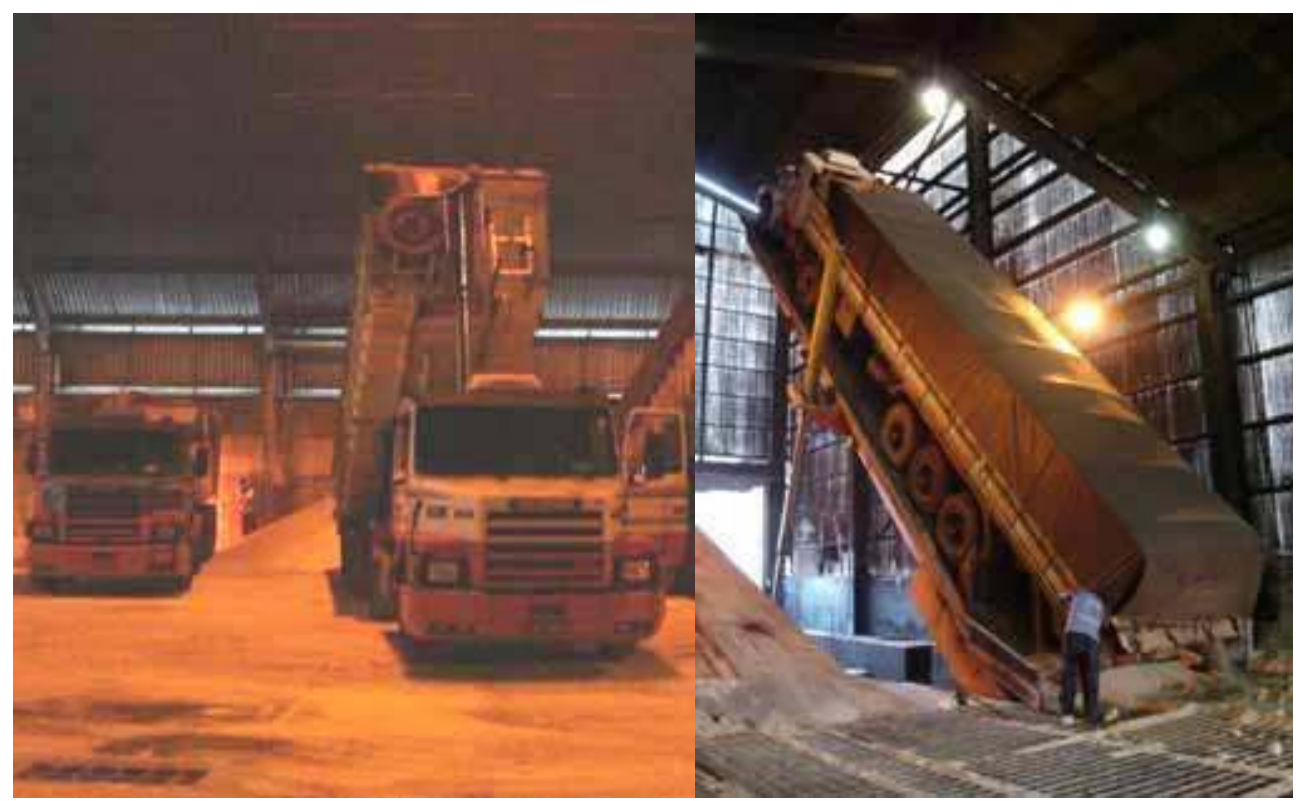

Fig. 2. Most common types of truck: a) Dump truck; b) Bulk truck tipped by a hydraulic tipping bridge.

This theft is possible due to the large size of the port area, where many companies are located together. Another fact that collaborates with theft is the distance between the 
warehouses and the $3^{\text {rd }}$ part weighting scales, seen in Fig. 3. There are two weighting scales external to the port area that serve the company when the number of trucks in the unload area is too high. Since the trucks have to move away from the port area to be weighed, it is easy for a trailer exchange to precede, for instance.

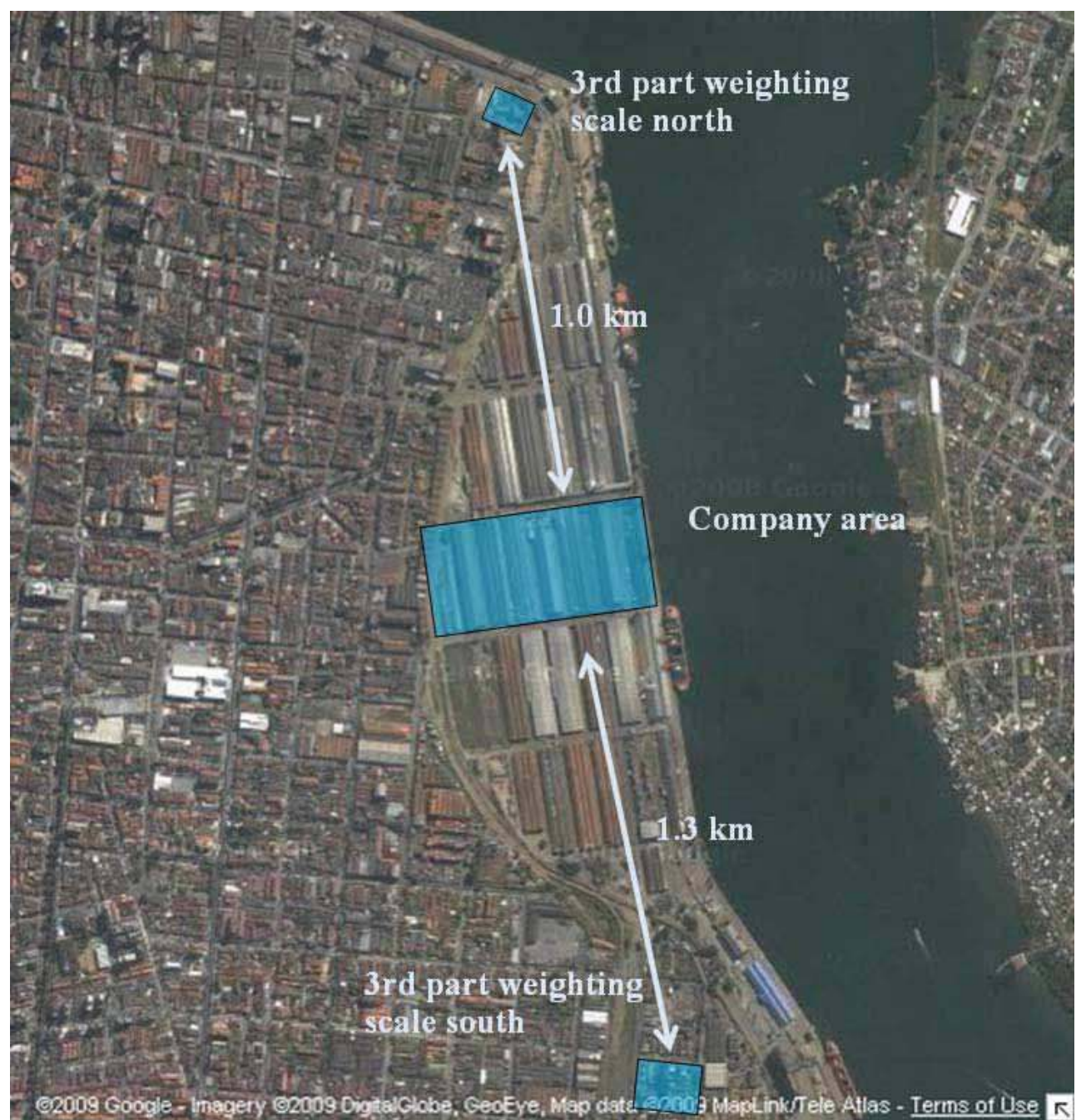

Fig. 3. The company area, and its distance to each external weighting scale.

The company unload area is $1 \mathrm{~km} \times 0.5 \mathrm{~km}$ long, and it has 6 warehouses, seen in Fig. 4 . Each of them can stock up to 30,000 tons of cargo; it is enough to completely load a bulk carrier. The company works with different types of products, so each type is stocked in one warehouse. Next to the warehouses, there is the company weighting scale. This scale has two weighting bridges, so it can weigh two trucks at the same time. Finally, the area has 
another small area built for administrative purposes and a datacenter building where all the servers are installed.

The solution developed and presented in this chapter, containing hardware and software to handle the trucks unload process in the port area is named Hunter. Its presentation starts with the introduction. Secondly the old process developed by the company studied will be presented. Later, the process and the analysis of possible technologies to be used to substitute the old process will be studied. After that, how the system was implemented is introduced, starting from the equipment and going through the software. Finally, the results, conclusion and future work are presented.

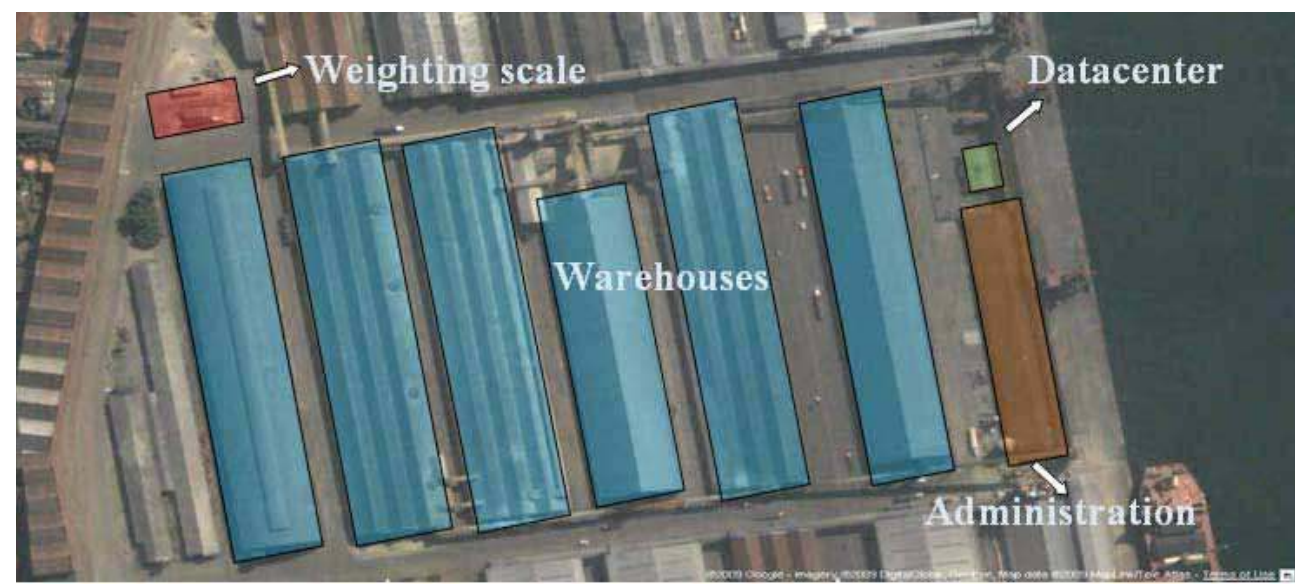

Fig. 4. Company unloading area.

\section{Related Works}

Nowadays, there are many works related to the use of RFID to control many types of processes. One relevant work is related to the pharmaceutical supply chain (Yue et al., 2008). The author proposes a new framework to develop a RFID system and applied it to the pharmaceutical process. The contribution of this work is the method described using the Critical Success Factor - CSF - and the " $5 \mathrm{~W} 1 \mathrm{H}$ " method. Another work is related to the process control of patient discharge process (Jiao et al., 2008). A re-engineering process is described, based on the Business Process Reengineering - BRP - methodology. The focus of the system is to automate human tasks, reduce data errors and collect data in real-time. The system was tested using simulation tools. Another work describes the application of the RFID to control attendance of students in classes (Qaiser \& Khan, 2006). It describes a system to track students and automatically detect their presence in the classes and measure the time that the student stayed in the classroom.

The postal service is a highly complex logistic company, and there is a relevant work describing the use of RFID for this type of service (Park et al., 2007). Here the integration of the managing system with PDAs is presented, as well as the description of the messages exchanged by the PDA and the managing system. The author creates a real test bed to 
measure the operationality of the system, containing RFID readers, PDAs and all the other equipment necessary to validate it.

Finally, another work describes only the system to handle the RFID events in a process control application (Hu et al., 2008). Many software approaches can be used to implement a RFID middleware and an event handling system. In this paper, the author used a hierarchy system with three layers using Petri nets.

All the works mentioned present software models, process approaches or methodologies to assist real RFID implementation. The difference is the fact that the Hunter system is the result of a real implementation case, described in this chapter, which started in 2005 and has been used by the company for over 3 years.

\subsection{Old Process}

A good related work reference is the solution that the company adopted previously to control its process. All the solution was based on barcode labels, barcode readers, a paper document called Minuta, from the Portuguese word for protocol, and the employees in charge of the unload process. This protocol has an ID, and it is a primary key for a management system called SSA, the function of which is to handle all the trucks unload. SSA is basically a client-server application that interacts with barcode readers and weighting scales to automatically capture the values without manually input.

The company defined two positions responsible for the unload operation: security inspector, or simply inspector, and the logistic supervisor, or simply supervisor. The inspector handles the truck information and the supervisor is responsible for the cargo information. They are both preset at all places in the unload area.

The Minuta is a piece of paper with all the information about the truck, the cargo and the service order, seen in Fig. 5. It will be named protocol to keep the text more readable.

When the truck arrives at the weighting scale, the driver gives the protocol to the inspector. The latter compares both the license plates of the tractor unit and trailer, the number of wheels and spare wheels, the departure place and some other information about the truck. If all the data are in accordance with details of truck, the inspector gives the protocol back to the driver and allows the truck to get inside the weighting scale to get the full weight measurement. Inside the weighting scale, the supervisor gets the protocol to consult the service order and the protocol id in the SSA, checking if this protocol is not fake or duplicated. If the system returns that it is a valid protocol, the supervisor captures the truck full weight and inserts it into the SSA database. After the weighing, the supervisor writes the current time in the protocol, gives it back to the driver and forwards the truck to one of the unloading places in the area. The selection of the unloading place is based on the type of the truck, type of the cargo and line size of the unloading places.

If any of the validation steps in the task of getting the full weight detects any discrepancy from the protocol, the truck is forwarded to the waiting area and the divergent data is checked with the location where the truck got the cargo. Some common problems detected are mostly related to the wrong typing of the license plate.

After the truck leaves the weighting scale, it proceeds to the line of the unload place previously assigned. Arriving in the line, the unloading area inspector gets the protocol again and double checks all the same truck information checked in the weighting scale. If all the information is correct, the inspector pastes a barcode label with his unique id merged with an incremental number, gives the protocol back to the driver and the truck gets inside 
the designated unloading place. There, the supervisor gets the protocol again and compares the type of cargo written in the protocol with the cargo inside the trailer. If all the data are in accordance with that information, the supervisor authorizes the beginning of the cargo unload. After the end of the unloading, the operator pastes another barcode label on the protocol with his unique id and another incremental number, writes the current time information on it and gives the protocol back to the driver.

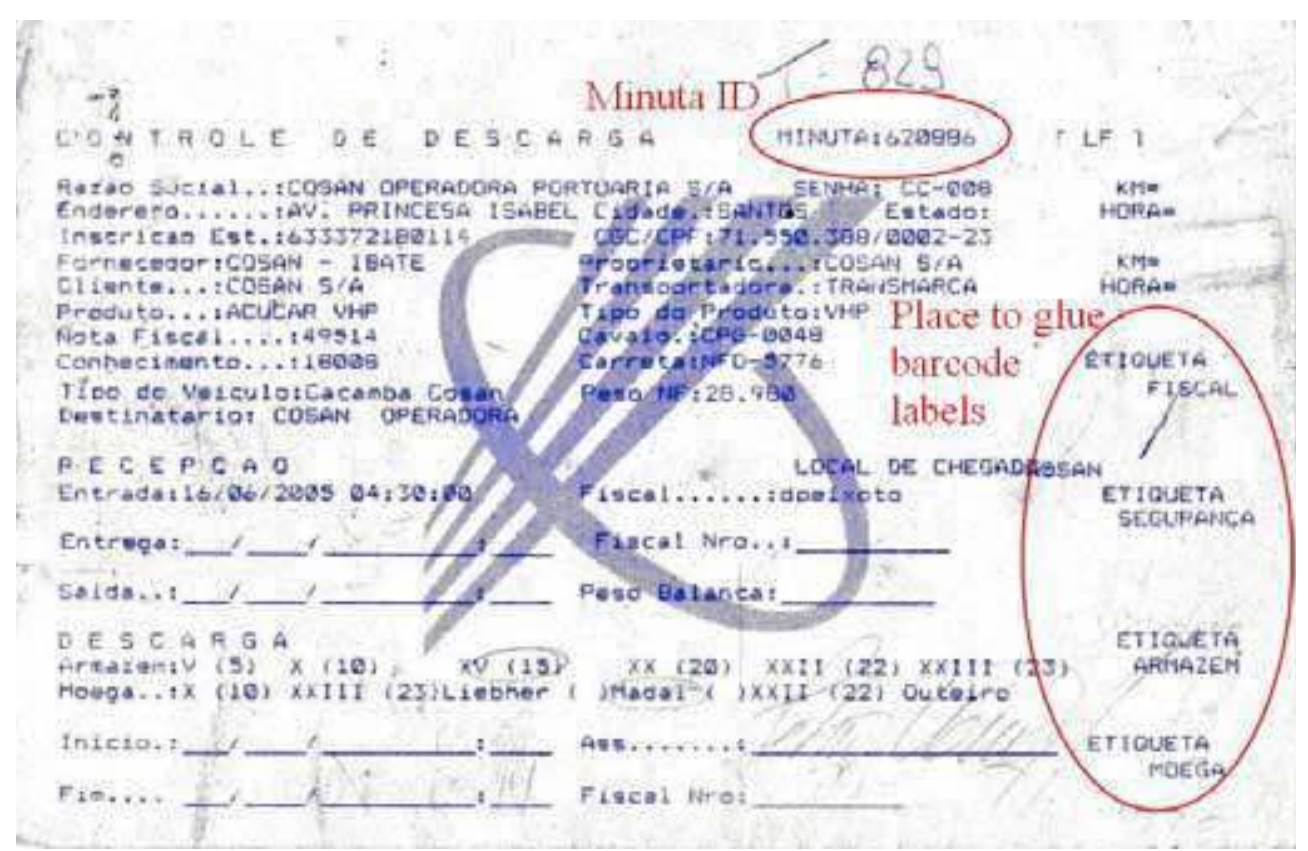

Fig. 5. A scanned copy of a Minuta, showing where its ID number is and where the barcode labels are pasted.

Here, as with the full weight procedure, if any of the validation steps detects a mismatch, the truck is forwarded to the waiting area. It keeps there until the point of discrepancy is solved. In most cases, the driver moves the truck to the wrong line, and it is detected by the inspector that forwards it to the correct line. If, for any reason, the truck conducted a partial cargo unload, it will be forwarded to another unloading place to repeat the procedure after the problem is solved.

When the truck is released, it has to go to the weighting scale again to proceed to the empty weight task. Arriving there, the truck repeats the same procedure conducted during the full weight task, with small differences. The driver gives the protocol to the inspector, who checks the data again and releases the truck to get inside the weighting scale. Inside it, the driver gives the protocol to the weighting scale operator who reads the barcodes using a barcode reader controlled by the SSA. If all the barcodes are accordance with previous information, the operator captures the empty weight and writes the current time information in the protocol. At this moment, the total cargo weight is calculated. If the difference between the calculated weight and the protocol declared weight is more than $5 \%$, 
the driver has the option to go back to any unloading place to proceed the scrapping and repeat the whole unload task. This weight difference happens because sometimes the product gets glued in the trailer wall. If the weight is within this tolerance, the driver gets his payment approved.

At the end of the day, all the protocols data are entered in the SSA manually. The information about the cargo weight and the unload place allows the software to calculate the amount of product unloaded for each type of product and how full the warehouses are. Finally, the timing data recorded in the protocol provides statistic information to measure the performance of the working team in each unloading place.

This solution has a weakness; it is based mostly on human intervention. It is totally dependent on the inspector and on the supervisor to keep the system trustable. Hence, an easy way to compromise the whole system is subverting these two people. For instance, a corrupt driver bribes both inspector and supervisor of a specific unloading place. Each person gives one of their barcode labels to the driver who pastes the labels on the protocol and fulfills the time fields with fake information. After that, the driver sells the cargo to a second company and unloads it there. Then, the driver goes to the weighting scale of the first company, with the trailer empty and the protocol fraudulently fulfilled with the barcode labels pasted. Finally, the driver performs the entire empty weight task without any problem and gets his payment. Thus, if the overall bribe paid to the inspector and supervisor is less than the amount paid by the second company for the cargo, the driver will make money with the fraud.

\section{Study on the Process and System Proposal}

As seen in the previous section, the solution adopted by the company is highly human dependent and has its weak points. The main issue is that the system has to be the least human dependent as possible. Therefore, to attain this objective, some pervasive approaches were studied and adopted.

So as to make the system less human dependent, it is necessary to remove the point of decision from the inspectors and operators. One critical point, for instance, is the truck entrance being informed by the inspectors, and the supervisor informing when the truck finishes the cargo unload. A solution to get this data automatically is to use a tracking device to monitor the truck in the unload area and to create virtual zones to detect which unload place the truck goes to, how long it takes to go from one place to the other and how many minutes it parks in each place.

Some technologies can be used to track vehicles; three were selected to be compared in this project based on the availability of the market and its cost: a) Global Positioning System GPS; b) Real-time Location System - RTLS; and c) Radio Frequency Identification - RFID (Guillemette et al., 2008). They will be explained and compared one by one further on.

Besides the system tracking functionality, some other features need to be added to the solution in order to meet the company needs. The license plate validation in each task of the process is one example of functionality the system must have. This can be done by using cameras and one optical character recognition - OCR - software. Yet, the company refused this technology arguing that the heavy dust present in the environment when the trucks unload the cargo will block the camera, compromising the capture of the license plate image. Thus, the decision was to keep the job of the security and operators just giving them 
a PDA to only conduct some of the confirmations they did in the old system. In this way, when the truck gets inside the unload place, the PDA software automatically presents the truck information and license plates, together with a confirm and refuse buttons. To increase the security of the solution, digital cameras were attached to the PDAs. These cameras are used to take pictures of the license plates and of the truck while it is unloading for further auditing. Since there is still dust, it is not a problem because the person who is handling the PDA can quickly clean it with his hands.

\subsection{GPS}

The first technology studied was the use of a GPS-based device to get the absolute latitude and longitude of the trucks along the unloading area. This device will be fixed in the trailer the moment the truck arrives at the weighting scale to capture its full weight. This GPS device will acquire the absolute position every second and transmit it via Wi-Fi or any other non-standard radio technology.

The problem in the use of this GPS device is related to the loss of satellite link when the truck gets into a closed environment. Since the GPS technology needs a clear vision of the sky, the GPS device will not be able to update its position when the truck gets into the warehouse and the unloading places (Jardak \& Samama, 2009).

A project approach to solve this problem is the use of a differential GPS - DGPS - installed inside the unloading places.

This first technology, using the GPS device attached to the truck trailer, will demand a full Wi-Fi coverage of the unloading area, a stock of GPS devices enough to be attached to all trucks in the unloading area at the peak time, and some DGPS equipment to be installed inside the unloading places to correct the positioning.

This option has the advantage of using a reliable technology such as GPS maintained by the American Department of Defense.

\subsection{RTLS}

The second option uses an emerging technology used to track objects inside closed areas called RTLS. This technology works based on a similar GPS principle called antenna triangulation.

To implement this approach, every square meter of the unloading area needs to be covered by at least three RTLS readers, which, in this case, will use Wi-Fi access points. In addition, one RTLS tag will be attached to each trailer, similarly to the first approach.

Briefly, this approach will need the same number of trailers attached to equipment as in the GPS approach, and the triple number of Wi-Fi APs.

\subsection{RFID}

Finally, the last option uses the RFID technology. Present RFID systems consist basically of four components: an electronic tag, a reader, an antenna, and application software to process the data. When a tag approaches the antenna, the latter sends a signal to the reader with the tag identification. The reader receives the signal, and, through a RS232 interface or an Ethernet port, the information is sent to a computer executing the software. This software is normally a middleware application that processes the data packets and sends them to an end-user application or a database (Kim \& Kim, 2006). 
RFID tags can be active or passive. The active ones are self fed by an internal battery and the passive ones are fed with the energy from electromagnetic waves sent by the reader. Regarding passive tags, the reading ranges vary between $5 \mathrm{~cm}$ and $10 \mathrm{~m}$. For the active tags, since they have an internal battery, the reading range can reach distances in the order of 200 meters. Active tags can be connected to temperature, tamper and movement sensors, among others. For this reason, the active tags technology was chosen for truck access control in warehouses and unloading area. In this case, the reader studied operates in the UHF frequency range, at $433 \mathrm{MHz}$ to transmit data and at $915 \mathrm{MHz}$ to receive data from the tag, with 80 -meter reading range.

The implementation of this technology will demand one RFID tag attached to each truck, as in both approaches already mentioned. Each unload area entrance and exit will need a RFID reader installed, as well as the weighting scale.

\subsection{Best approach}

In order to choose the best technology, all of them were inserted into a table to facilitate the comparison, seen in Table 1.

The criterion chosen was the final cost of the whole solution. In this context, the RFID was the best technology.

\begin{tabular}{l|l|l|l|l|}
\cline { 2 - 5 } Approach & $\begin{array}{c}\text { Trailer Attached } \\
\text { Device Cost }\end{array}$ & $\begin{array}{c}\mathbf{N}^{\circ} \text { of Wi-Fi } \\
\text { APs }\end{array}$ & $\begin{array}{c}\text { Cost in Network } \\
\text { Infrastructure }\end{array}$ & \multicolumn{1}{|c|}{$\begin{array}{c}\text { Additional } \\
\text { Equipment }\end{array}$} \\
\hline GPS & Very Expensive & Regular & Regular & DGPS \\
\hline RTLS & Regular & Large & Great & Wireless Switch \\
\hline RFID & Regular & Small & Small & RFID Readers \\
\cline { 2 - 6 }
\end{tabular}

Table 1. Comparison between the different tracking technologies.

\section{Implementation and Implementation}

The solution containing the RFID readers, RFID tags, PDAs, Wi-Fi access points, infrastructure and the software that handles all the data between these devices was named Hunter, an acronym for Hybrid Unified Tracking Environment. Hunter implementation was divided into two fronts: One developing the software and the second handling the network infrastructure and the installation of the equipment.

\subsection{Infrastructure}

To cover all the entrances and exits of every unload place and weighting scale, 32 readers were necessary. Due the different range of distance between each equipment, different technologies were used to connect them all. The warehouses equipment was at a maximum distance of $1 \mathrm{~km}$ from the server, so it was not possible to use Ethernet cables. Since the pipes infrastructure was already available, it was connected using optical fiber. The weighting scales were farther than the warehouses, and these scales were in an area outside the port, in the middle of the city. Based on that, a fiber link to connect both places would be very expensive. Thus, the choice was to use a point-to-point radio link to connect the weighting scale into the equipment network. 


\subsection{The Padlock Tag}

Working as an electronic seal, a tamper sensor was attached to the tag. This sensor accuses "ok" status if its terminal is placed in short circuit and "violated" if the terminal is in open circuit. This feature allowed the development of a tag model that could be fixed at a truck dump-cart. If a tag removal attempt occurs, the opening sensor will detect it and send a violation signal to the nearest reader. Several readers were installed at the unloading area, in case any anomaly occurred within the normal procedures (for example, the truck enters some unloading area and does not unload or violates the tag) an alarm will be presented in the weighting scale operator's screen when the truck arrives there, or in the PDA of the unload operators.

The main difficulty found in the tag design was the great difference between trucks used by the company which made it difficult to develop a single tag that could be fixed in each of them. To solve the problem, the tag was designed be similar to a padlock that can be fixed to any bar up to two inches thick. Therefore, it was possible to fix a tag in multiple parts of the truck. The violation detection of a tag is made by a small switch; when the lock is closed, this switch is pressed and when the lock is opened, the switch is released. This switch was connected by two wires to the tag circuit violation terminals sensor. The padlock RFID tag can be seen in Fig. 6 and Fig. 7.

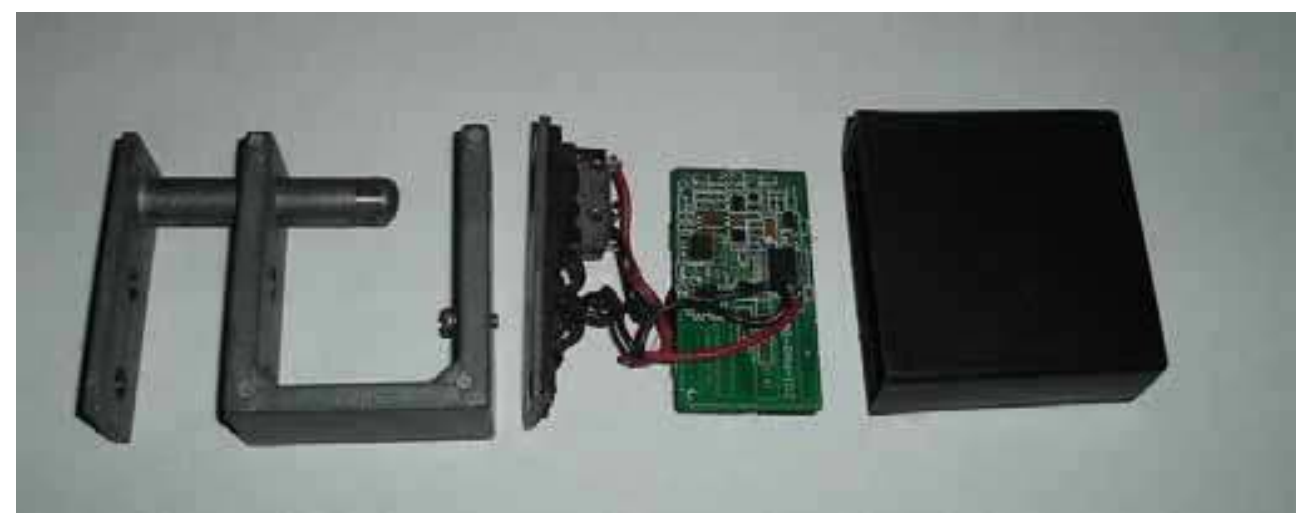

Fig. 6. Active tag in a padlock shape with opening detector disassembled.

\subsection{Equipment Setup}

The rule adopted to install readers was: a reader was installed at each entrance and exit. For example, at the weighting scale platform, two readers were installed, one at the entrance and another one at the exit. At the unloading warehouses, one reader was installed at each entrance/exit gate. All readers were connected by Unshielded Twisted Pair (UTP) cables Cat 5e. Warehouses and weighting scales were connected by multimode optical fiber cables, thus building a 1 GBPS local area network between all devices and the server which executes the managing application.

In sum, a reader was installed at each truck entrance/exit and an Ethernet point was installed near each reader to put it into the equipment local area network. All readers were connected to the server which executes the managing application. Each truck passage through a reader generates a data packet to the server with information containing the tag 
number, electronic seal status, timestamp and the number of the reader that received the packet.

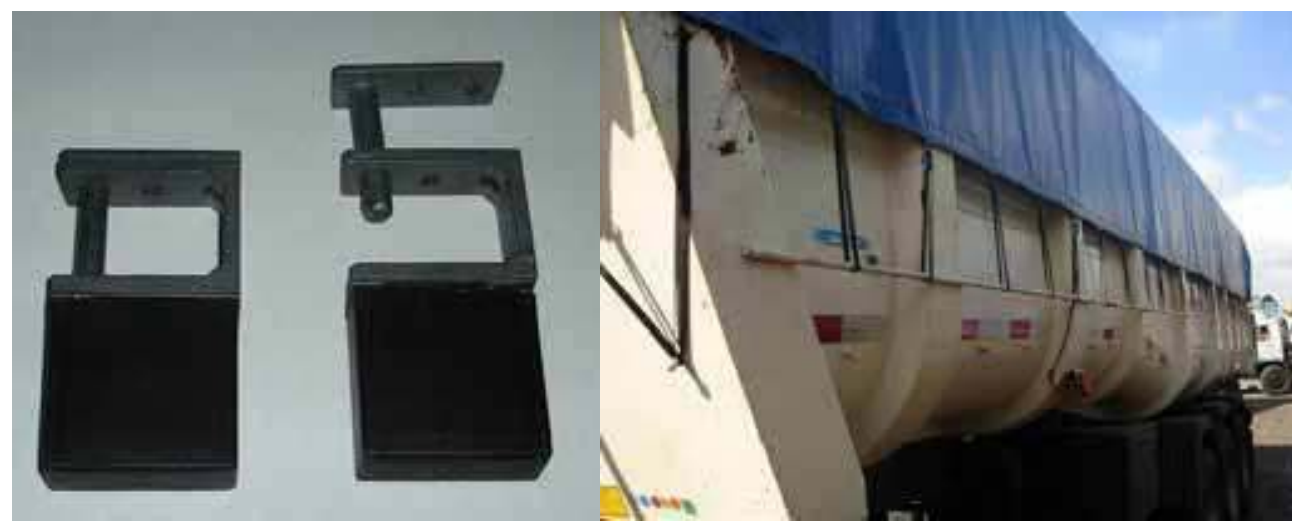

Fig. 7. a) Padlock tag assembled, opened and closed; b) One type of trailer showing the iron bar where the padlock tag is attached.

\subsection{Software}

The managing application, called Hunter - Hybrid Unified Trekking Environment, controls the unloading process information over the monitored vehicles. Every truck is treated by the system as an event queue to be executed. Inside the system, every truck passage through an entrance/exit is an event. Full weight, unload and empty weight are examples of events to be executed by the truck. Thus, in the system, each truck has a state within a finite state machine. Through received events by that truck, it registers exactly at which stage of unloading flow the truck is in real-time. In other words, the trucks in movement inside the area are treated as independent threads in the system, which is initiated when its tag passes the weighting scale to capture the full weight, generating the first event. When these threads are created, a task queue at the data base is created as well, with all the tasks the truck must accomplish along the process, such as: weighting scale entrance for full weight, full weight capture, weighting scale exit, unloading area entrance, PDA operator confirmation, etc. While the truck moves and generates the events, these are interpreted by the system and converted into its respective tasks, and thus they are removed from the task queue and inserted in the journal table. In case the tasks orders are not obeyed, an alarm is generated on the system operator screen.

The system graphical interface shows the number of trucks on each state of the unloading process, seen in Fig. 8. The trucks in the lines between each state are shown as well. There are thus five states presented in the interface: Full weight capture, Unload line, Unloading process, Empty weight line and Empty weight capture. Through the graphical interface, it is possible to see the trucks that are at this state and how long they are there.

The application was totally developed in Java, being a part of it monolithic and a part in form of a web-base application. It is executed by a Tomcat application server and connected to an Oracle 9i data base with features of advanced task queue. As different technologies are used, the managing application was developed with four great modules, presented in Fig. 9 (Chen et al., 2003). 
The first system layer, called Device Management Subsystem (DMS) is the layer responsible for the connection between the physical device (RFID readers, PDAs, etc) and the system engine for process control, times and alarms, here called Core. Inside the DMS, there is an application part (DMSApp) and a servlet part (DMSServer). The application keeps waiting for a package at the serial port or at the Ethernet connections, and when it receives data sent by any device, it interprets the information and generates an extensible markup language XML - message with the content that is transmitted to the DMSServer. When The DMSServer receives this XML, it consults the database table where the information about the devices positioning is. After that, it generates a XML of a higher level to be sent to Core, with information on the name of the place the tag was read and with details of the truck which has the tag. DMS is a middleware, concurrently connecting different devices in the system which allows new devices to be connected to the system without any change in the source code of the application.

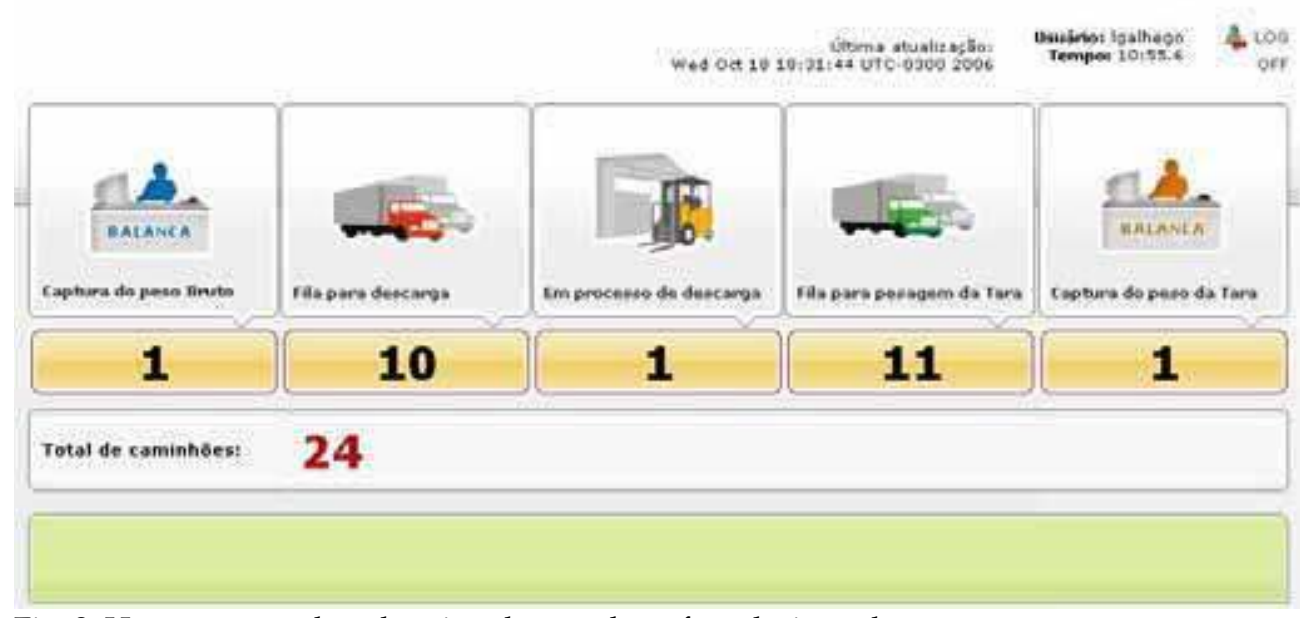

Fig. 8. Hunter screenshot showing the number of trucks in each state.

A difficulty was found in the calibration of the power emitted by the RFID equipment to obtain readings of all tags. As explained before, the dump-cart material influence and the area where the tag is placed significantly modify the reading range. Since there are trucks with wooden and steel dump-carts, the calibration was made for the worst case scenario, increasing the emitted power beyond necessary. This increase brought a few problems, such as continuous readings and anticipated readings. For example, depending on where the tag was placed in the truck, the weighting scale exit reader could read the tag constantly during its weighting before it left and, in other cases, the scale entrance reader kept reading during its weighting. To avoid this problem, a series of filters was inserted at the DMS so the real information would arrive clearly to Core. For these cases in which the entrance reader constantly reads the truck tag, an anti-repetition filter was developed, sending only the first entrance reading and ignoring the following at a certain interval of time. In the case of exit reader, a delayer filter was developed, so it would only send the last exit reading, ignoring 
the first ones. Therefore, the filters prevent the same entrance event from being sent twice and also prevent the exit event from being sent prematurely.

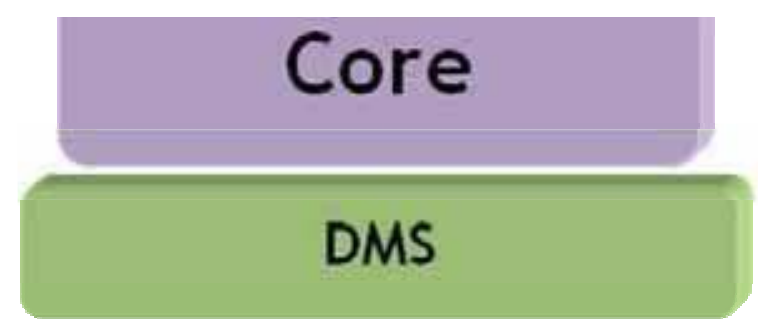

Fig. 9. Managing applicative architecture

The Core has a servlet that receives the XMLs from DMSServer and inserts them into the data base. When this insertion is made, the table where the information is stored has a trigger that initiates all the data analysis process, such as the alarms that can be generated due to this event or updates on the truck state in the unloading process. Connected to Core, there are two other modules, the graphical interface and the legacy integration systems. The first one is responsible for presenting information on the screen, such as alarms, navigation maps and reports generation. The second module is responsible for sending information to the company legacy system, updating the warehouse free space, linking timing information and alarms generated to the company corporative systems.

All the communication between system modules is made through XML messages and all the system graphical interfaces are web-based, allowing their access from any computer without the need of specific application installation, just a conventional web browser. The integration of the managing application with the company corporative systems is made through recorded text files containing the XML messages in a shared directory.

\section{Problems and Solutions}

The place where the tag is attached on each truck may vary depending on the truck dumpcart, and its material can vary between wood and steel, as well. These variations can influence the sensitivity of the tags, causing undesired tag readings outside the calibrated reader reading range (Foina et al., 2006). These readings happen when the truck is crossing the warehouse entrance, causing unexpected alarms and making the system call the security team unnecessarily. To solve it, two resources were used.

The first is the use of a directive antenna in the place of the omnidirecional antenna normally used in the active tags readers. The directive antenna used in the experiments was a patch antenna with the characteristics presented in Table 2. 
This antenna will amplify the signal received in its directive lobule, sending a stronger signal to the reader in this case. However, if the signal is received outside its directive lobule, the reader will receive a weak signal (Balanis, 1997).

The second resource used is a feature of the active readers that allow measuring the signal strength received by the reader. This feature is called RSSI - Received Signal Strength Indication. In the equipment chosen, the RSSI is a rate from 0 to 255 , where a higher value means a stronger signal. Plotting a graphic and placing the reader in mark 0 of the distance axis, it is possible to see the RSSI variation related to the tag position.

\begin{tabular}{ll}
\hline Specification & Value \\
\hline E-Plane Beamwidth & 65 degrees at 3 dB \\
Frequency Range & $902-928 \mathrm{MHz}$ \\
Gain & $7 \mathrm{dBi}$ \\
H-Plane Beamwidth & 65 degrees at $3 \mathrm{~dB}$ \\
Impedance & 50 Ohms \\
Maximum Input Power & 1 Watt \\
Polarization & Circular \\
VSWR & $1.5: 1$ \\
\hline
\end{tabular}

Table 2. Directive antenna specification

Fig. 10 and Fig. 11 show the RSSI rate for each position of the tag in relation to the reader: the first using an omnidirectional antenna and the second using the directive antenna. The distances vary from $-6 \mathrm{~m}$ to $4 \mathrm{~m}$. The reader is positioned in mark 0 in the scale of both graphics. In Fig. 10, the directive antenna is placed in position $-1 \mathrm{~m}$. The difference in the curves of Fig. 10 and Fig. 11 are noticeable. Fig. 10 shows that the RSSI index slowly increases with the position until the tag reaches the reader. After that, it starts decreasing symmetrically. In Fig. 11, the RSSI rate increases rapidly when the tag moves to the front of the directive antenna, drawing an asymmetric curve.

The RSSI rate can present some oscillations due to signal reflections and noise (Zhao, 2006). For this reason, the middleware can analyze the RSSI by averaging a certain number of values using a five-period moving average. This filters out rapid variations of the signal to be presented, so each figure has two curves; the first is the signal received and the second is the averaged values.

The solution for the undesired tag readings errors is the joint use of the RSSI measurement and a directive patch antenna for the reader. In this case, RSSI will be used to inform the software if the tag is behind or in front of the antenna. The directive antenna was installed behind the reader, facing the warehouse door, approximately 1 meter away (Fig. 12). When the tag is close to the door, but behind the antenna, the RSSI index will be very low because the tag is not in the main lobule of the antenna. When the tag is positioned between the door and the antenna, the RSSI index will be much higher than if measured in the same position with an omnidirectional antenna. This enables the middleware to make the decision of whether or not to grant access into the warehouse. 


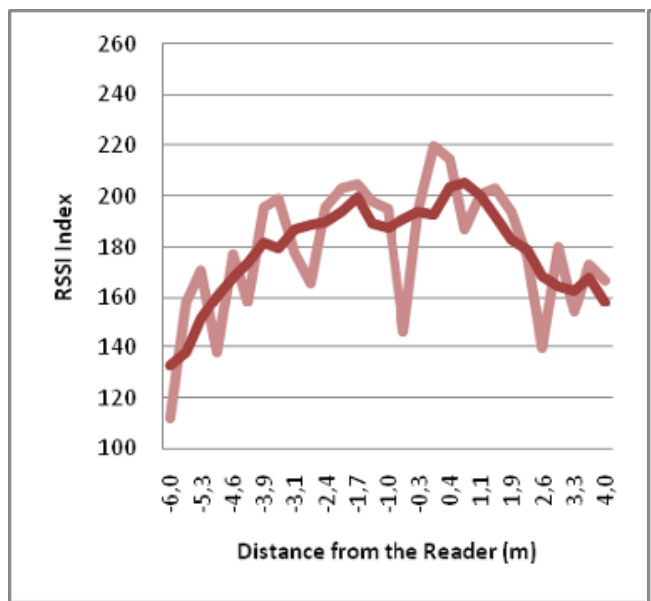

Fig. 10. RSSI changes based on the distance with an omnidirectional antenna.

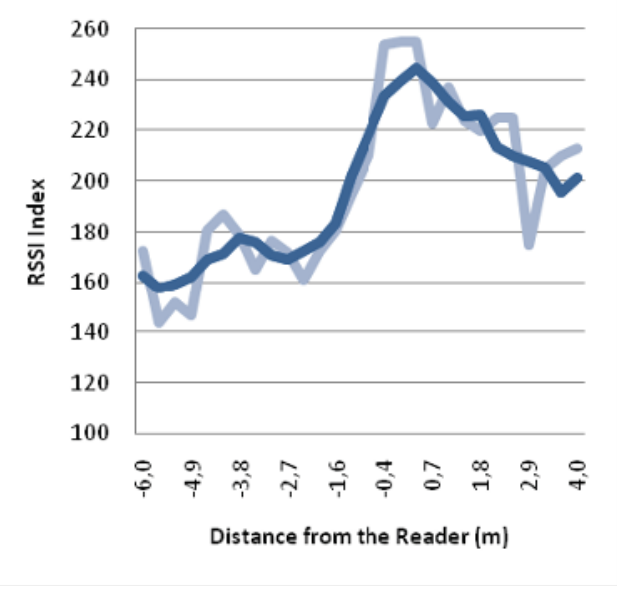

Fig. 11. The changes in the RSSI rate using a directive antenna 1 meter away from the reader.

Fig. 12. Aerial representation of the reading zone of both approaches, a) using the omnidirectional antenna and $b$ ) using the directive antenna.

\section{Results and Conclusion}

The use of the system allows the company to have a tighter control of its operation due the real-time information. In this way, the company logistic department can keep an almost constant number of trucks in the unload area, avoiding long lines and trucks shortage, as seen in Fig. 13. 
Despite the difficulties in installing a wireless network and an optical fiber network in old warehouses, and placing sensitive radio frequency equipment exposed to weather hazards, it was possible to successfully install all the equipment and to calibrate it. The PDA and server applications behaved correctly when up to 200 trucks were simultaneously circulating in the area.

With the additional information generated by the system for the logistic department about the weight, unload time statistics, and the number of trucks in real time at each stage of the unloading process, it was possible to optimize the trucks line, reducing the wait in line and therefore, reducing the average time of the unload process from 50 minutes to 30 minutes. The installation of RFID padlock tags in the trucks reduced load theft by $60 \%$ in the port area and allowed the security team to find out points of vulnerability in the previous system and to detect most of the truck drivers corrupted by the theft group.

In conclusion, Hunter showed the expected results after one year of close observation. With the reduction in the company yearly loss, the project payback occurred in less than one year.

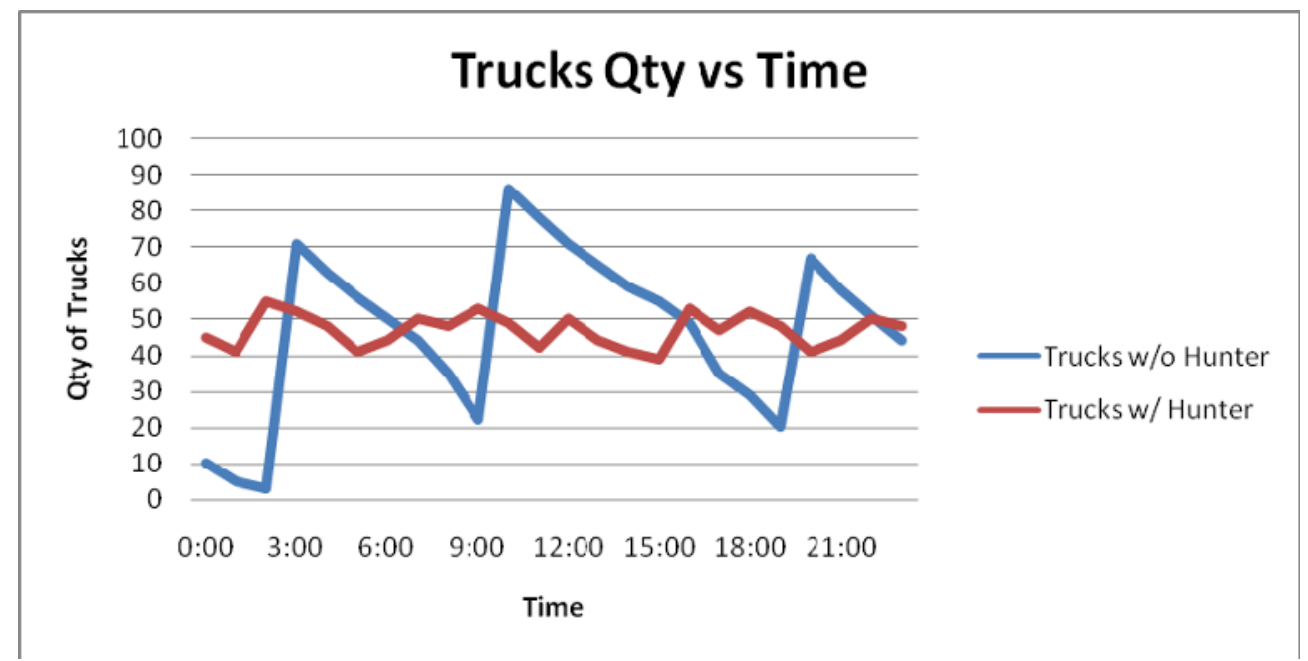

Fig. 13. Number of trucks in the unloading place for each hour of a regular day.

\section{Future works}

The Hunter system can be applied to many different applications and processes. For instance, it can be used with passive tags to control automated manufacture lines, supply chain pallets and forklifts. The application will control if the device goes through all the phases in the manufacturing line, from the beginning to the expedition. It can control who the forklift operator was and how long he took to move the products from one place to other. The product will thus be tracked, supplying information about the storing and movement time, and the forklifts will be tracked as well, supplying information about the efficiency of the forklift operator and generating alarms if he does something wrong. Therefore, as future work, Hunter will be tested in a different process to check its flexibility. 


\section{References}

Almanza-Ojeda, D. L.; Hernandez-Gutierrez, A.; Ibarra-Manzano, M. A.; (2006). Design and implementation of a vehicular access control using RFID, Multiconference on Electronics and Photonics, pp. 223 - 225. 2006.

Balanis, C. A.; (1997). Antenna Theory: Analysis and Design, John Wiley \& Sons, USA, second edition, pp. 86-88.

Blythe, P.; (1999). RFID for road tolling, road-use pricing and vehicle access control. IEE Colloquium on RFID Technology, Ref. No. 1999/123, pp. 8/1 - 816, 25 Oct. 1999.

Chen, S.; Gulati, S.; Hamid, S.; Huang, X.; Luo, L.; Morisseau-Leroy, N.; Powell, M. D.; Zhan, C.; Zhang, C; (2003). A three-tier System Architecture Design and Development for Hurricane Occurrence Simulation. Proceedings of Information Technology: Research and Education, pp. 113 - 117, 11-13 Aug. 2003.

Foster, P.R.; Burberry, R.A.; (1999). Antenna problems in RFID systems. IEE Colloquium on RFID Technology, Ref. No. 1999/123, pp. 3/1 - 3/5, 25 Oct. 1999.

Foina, A. G.; Barbin, S. E.; Ramirez Fernandez, F. J.; (2007). A New Approach for Vehicle Access Control using Active RFID Tags. Proceedings of Microwave and Optoelectronics Conference, pp 90-93, 29 Out. 2007.

Greenfield, A (2006); Everyware: the dawning age of ubiquitous computing. New Rider. ISBN 0321384016.

Guillemette, M. G.; Fontaine, I.; Caron, C. (2008). Hybrid RFID-GPS Real-Time Location System for Human Resources: Development, Impacts and Perspectives. Proceedings of the 41st Annual Hawaii International Conference on System Sciences, pp. 406-406, Jan. 2008

Jardak, N.; Samama, N. (2009). Indoor Positioning Based on GPS-Repeaters: Performance Enhancement using an Open Code Loop Architecture. IEEE Transactions on Aerospace and Electronic Systems, vol. 45, no. 1, pp. 347-359, Jan. 2009.

Jiao, Y.-Y.; Li, K.; Jiao, R.J. (2008). A case study of hospital patient discharge process reengineering using RFID. 4th IEEE International Conference on Management of Innovation and Technology, pp. 1342-1347, Sept. 2008.

$\mathrm{Hu}$, W.; Ye, W.; Huang, Y.; Zhang, S. (2008). Complex Event Processing in RFID Middleware: A Three Layer Perspective. Third International Conference on Convergence and Hybrid Information Technology, vol. 1, pp. 1121-1125, Nov. 2008.

Kim, T.; Kim, H.; (2006). Access Control for Middleware in RFID Systems. The 8th International Conference on Advanced Communication Technology, vol. 2, pp. 1020 1022, 20-22 Feb. 2006.

Ni, L. M.; Liu, Y.; Lau, Y. C.; (2003). LANDMARC: Indoor Location Sensing Using Active RFID. In IEEE International Conference in Pervasive Computing and Communications, pp. 407-415, 2003.

Park, J.-H.; Park, J.-H.; Lee, B.-H. (2007). RFID Application System for Postal Logistics. Management of Engineering and Technology, pp. 2345-2352, Aug. 2007.

Qaiser, A.; Khan, S.A. (2006). Automation of Time and Attendance using RFID Systems. International Conference on Emerging Technologies, pp. 60-63, Nov. 2006.

Raza, N.; Bradshaw, V.; Hague, M.; 1999. Applications of RFID technology. In IEE Colloquium on, pp. 1/1 - 1/5, 25 October, 1999. 
Tuttle, J.R.; (1997). Traditional and emerging technologies and applications in the radio frequency identification industry. Symposium on Radio Frequency Integrated Circuits (RFIC), pp. 5 - 8, 8-11 Jun. 1997.

$\mathrm{Xu}, \mathrm{G}$. (2008). The Research and Application of RFID Technologies in Highway's Electronic Toll Collection System. Wireless Communications, Networking and Mobile Computing 2008, pp. 1-4, Oct. 2008.

Yue, D.; Wu, X.; Bai, J. (2008). RFID Application Framework for pharmaceutical supply chain. IEEE International Conference on Service Operations and Logistics, and Informatics, vol.1, pp. 1125-1130, Oct. 2008.

Zhao, J.; Zhang, Y.; Ye, M.; (2006). Research on the Received Signal Strength Indication Location Algorithm for RFID System. International Symposium on Communications and Information Technologies, pp. 881 - 885, Oct. 2006. 


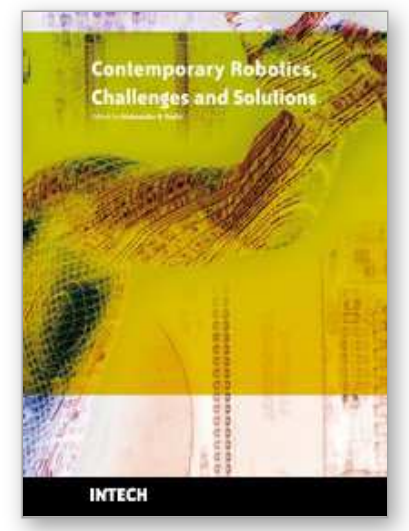

\section{Contemporary Robotics - Challenges and Solutions}

Edited by A D Rodi

ISBN 978-953-307-038-4

Hard cover, 392 pages

Publisher InTech

Published online 01, December, 2009

Published in print edition December, 2009

This book book is a collection of 18 chapters written by internationally recognized experts and well-known professionals of the field. Chapters contribute to diverse facets of contemporary robotics and autonomous systems. The volume is organized in four thematic parts according to the main subjects, regarding the recent advances in the contemporary robotics. The first thematic topics of the book are devoted to the theoretical issues. This includes development of algorithms for automatic trajectory generation using redudancy resolution scheme, intelligent algorithms for robotic grasping, modelling approach for reactive mode handling of flexible manufacturing and design of an advanced controller for robot manipulators. The second part of the book deals with different aspects of robot calibration and sensing. This includes a geometric and treshold calibration of a multiple robotic line-vision system, robot-based inline 2D/3D quality monitoring using picture-giving and laser triangulation, and a study on prospective polymer composite materials for flexible tactile sensors. The third part addresses issues of mobile robots and multi-agent systems, including SLAM of mobile robots based on fusion of odometry and visual data, configuration of a localization system by a team of mobile robots, development of generic real-time motion controller for differential mobile robots, control of fuel cells of mobile robots, modelling of omni-directional wheeled-based robots, building of hunter- hybrid tracking environment, as well as design of a cooperative control in distributed population-based multi-agent approach. The fourth part presents recent approaches and results in humanoid and bioinspirative robotics. It deals with design of adaptive control of anthropomorphic biped gait, building of dynamic-based simulation for humanoid robot walking, building controller for perceptual motor control dynamics of humans and biomimetic approach to control mechatronic structure using smart materials.

\section{How to reference}

In order to correctly reference this scholarly work, feel free to copy and paste the following:

Aislan Gomide Foina and Francisco Javier Ramirez-Fernandez (2009). Hunter - Hybrid Unified Tracking Environment, Contemporary Robotics - Challenges and Solutions, A D Rodi (Ed.), ISBN: 978-953-307-038-4, InTech, Available from: http://www.intechopen.com/books/contemporary-robotics-challenges-andsolutions/hunter-hybrid-unified-tracking-environment

\section{INTECH}

open science | open minds

\section{InTech Europe}

University Campus STeP Ri

Slavka Krautzeka 83/A

\section{InTech China}

Unit 405, Office Block, Hotel Equatorial Shanghai

No.65, Yan An Road (West), Shanghai, 200040, China 
51000 Rijeka, Croatia

Phone: +385 (51) 770447

Fax: +385 (51) 686166

www.intechopen.com
中国上海市延安西路65号上海国际贵都大饭店办公楼 405 单元

Phone: +86-21-62489820

Fax: $+86-21-62489821$ 
(C) 2009 The Author(s). Licensee IntechOpen. This chapter is distributed under the terms of the Creative Commons Attribution-NonCommercial-ShareAlike-3.0 License, which permits use, distribution and reproduction for non-commercial purposes, provided the original is properly cited and derivative works building on this content are distributed under the same license. 